\title{
SideBar: Videoconferencing System Supporting Social Engagement
}

\author{
Morten Esbensen, Paolo Tell, Jakob E. Bardram \\ Pervasive Interaction Technology Laboratory \\ IT University of Copenhagen \\ Rued Langgaardsvej 7, 2300 Copenhagen S, Denmark \\ Email: \{mortenq,pate,bardram\}@itu.dk
}

\begin{abstract}
Companies are increasingly organizing work in globally distributed teams. A core challenge to these distributed teams is, however, to maintain social relationships due to limited opportunities and tools for social engagement. In this paper we present SIDEBAR: a videoconferencing system that enhances virtual meetings by enabling social engagement. Through image analysis of the conference video feed, SIDEBAR tracks meeting participants in real-time. A personal tablet then allows each participant to identify and track other participants, to look up information about them and their local work context, and to engage in peer-to-peer chat conversations. We describe the motivation, design and implementation of SIDEBAR and report results from a preliminary evaluation, which shows that participants found SIDEBAR useful and easy to use. The paper concludes by providing three design guidelines for collaborative technologies supporting social engagement.
\end{abstract}

\section{INTRODUCTION}

Companies are progressively organizing work in globally distributed teams [1]. It has been recognized that such distributed collaboration is affected by new challenges, which, according to Herbsleb [2], can be ascribed to the absence or disruption of those mechanisms that in collocated settings naturally support coordination among practitioners. Examples of these mechanisms range from spontaneous engagement in conversation to visual clues conveying awareness capable of offering insights on, for instance, availability of team members as well as their current activity. Studies reflecting on the challenges of distributed collaboration (e.g., [3], [4]) agree on the view that physical distance is a factor of paramount importance that profoundly influences how people interact [5].

Social relationships and engagement are in particular negatively affected by geographical distance [6], [7]. Given for granted in collocated settings and often overlooked and underestimated in distributed arrangements, social engagements represent powerful facilitators capable of fostering successful collaboration [8], which have to be nurtured and encouraged not only by practices but also by technologies. Important aspects of social engagement, which have been investigated and for which direct dependences have been observed are: the feeling of group cohesion, which has been argued to be a facilitator for the effectiveness of communication technologies [9]; the feeling of connectedness, for which an impact on communication has been identified [10]; the awareness of remote team members and of their locations, which have been linked to communication patterns [11]; and, trust, which has been investigated from multiple angles and perspectives as systematically reported in [12].

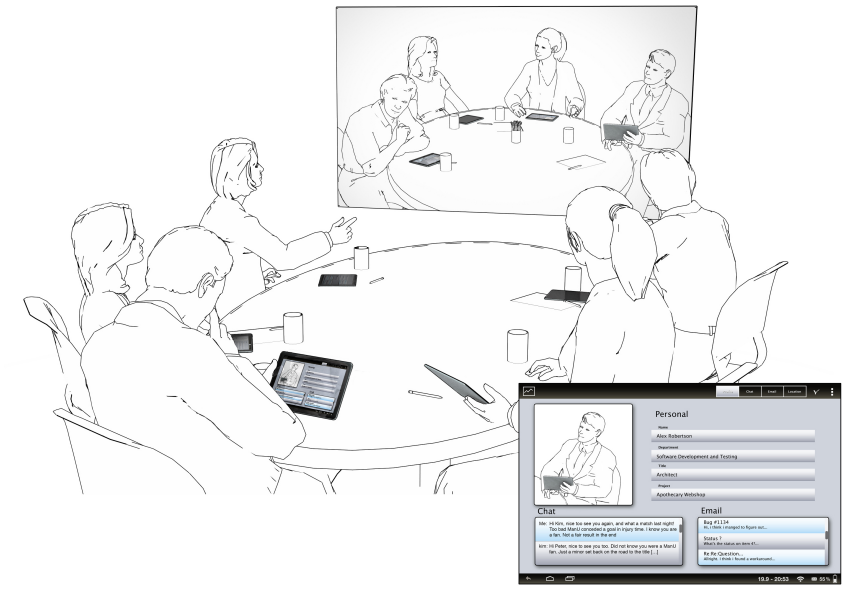

Fig. 1. SIDEBAR is a videoconferencing system supporting social engagement. This is achieved by face tracking in the video feed and by provide an interactive mirrored video feed on tablet computers, allowing users to recognize and seek information about each other, and engage in backchannel peer-to-peer chat conversations.

A core challenge in distributed collaboration is, therefore, how to support such social engagements. Collocated environments provide good conditions for social engagements to occur through shared physical spaces allowing for mutual awareness, close collaboration, shared experiences, and social encounters [5]. In distributed arrangements, however, actors have to commit to much more explicit work to cultivate such social aspects [13], especially when the social connections required to initiate these aspects of collaboration need to be established (e.g., visit to remote site and team building activities [14]).

To address the challenge of providing social engagements in distributed arrangements, we have designed SIDEBAR: a videoconferencing system with special support for social engagements. SIDEBAR incorporates face tracking into a videoconferencing system to provide an interactive mirrored video feed on tablet computers that allows users to recognize and seek information about each other and engage in backchannel peer-to-peer conversations.

In this paper, we provide an overview of videoconferencing solutions and then describe the SIDEBAR system. The more significant design decisions are discussed before detailing the architecture of the system. A preliminary evaluation of the system is also presented, which aimed at assessing the 
perceived usefulness and ease of use of SIDEBAR, as well as whether or not the system was perceived as a distraction. Finally, before concluding, we propose a set of guidelines for designing collaborative technologies with support for social engagement.

\section{BACKGROUND AND RELATED WORK}

Social connections are core to collaboration [8] but challenged in distributed arrangements. For example, Herbsleb and Mockus [7] have shown that the size of social networks in distributed arrangements is smaller as compared to collocated ones, and that the ability to recognize remote team members is more difficult for distributed team members. Similarly, it has been found that people tend to form groups with collocated colleagues rather than distant ones [15]. Social factors, however, are important in distributed arrangements in which the communication is affected by the feeling of connectedness [10] and of group cohesion [9]. Social connections and engagement, thus, are of much importance when designing tools supporting distributed collaboration.

Videoconferencing meetings are used extensively in distributed collaboration and are, in terms of media richness theory, the closest we come to collocated meetings and collaboration [16]. However, despite the richness of the video medium, a meeting supported by technologies still does not compare to a collocated face-to-face one [17]. Therefore, a significant body of research has focused on bringing the feeling of 'sitting together' to the video meeting by improving the videoconferencing technology to include information intrinsic to the collocated meeting such as eye-contact [18] and non-verbal cues [19]. Such information can be valuable in distributed collaboration by increasing the social awareness between team members [20].

The setup of videoconferencing equipment has been shown to affect different aspects of participants perception of each other. Using a video setup that captures both face and upper body have been shown to have a positive result on trust and empathy in groups as opposed to only capturing the face [21]. Another study of trust in different group-to-group videoconferencing setups suggests that a combination of personal displays and individual streams of each participant contribute to a higher level of trust development [22], and that the perception of proximity in videoconferencing is linked to the zoom of the camera [23].

GAZE-2 [18] is a group video system that supports eyecontact transmission. Using several cameras and an eye-tracker per setup, the system ensures parallax-free transmission of eye contact, by choosing the camera the user is looking at. eyeView [24] is a videoconferencing system that leverages gaze direction. Using eye tracking to resize individual video windows based on looking behavior, eyeView keeps focus on the current speaker while keeping an overview of all participants though scaled down videos of them. These systems are examples of technologies, which include eye-contact [25] or 3D-experience [26] in traditional videoconferencing setups.

Other approaches to enhance videoconferencing have focused on creating a more immersive experience. MAJIC [27], for example, uses large curved, semi-transparent displays with cameras placed behind them. This setup allows for life-size

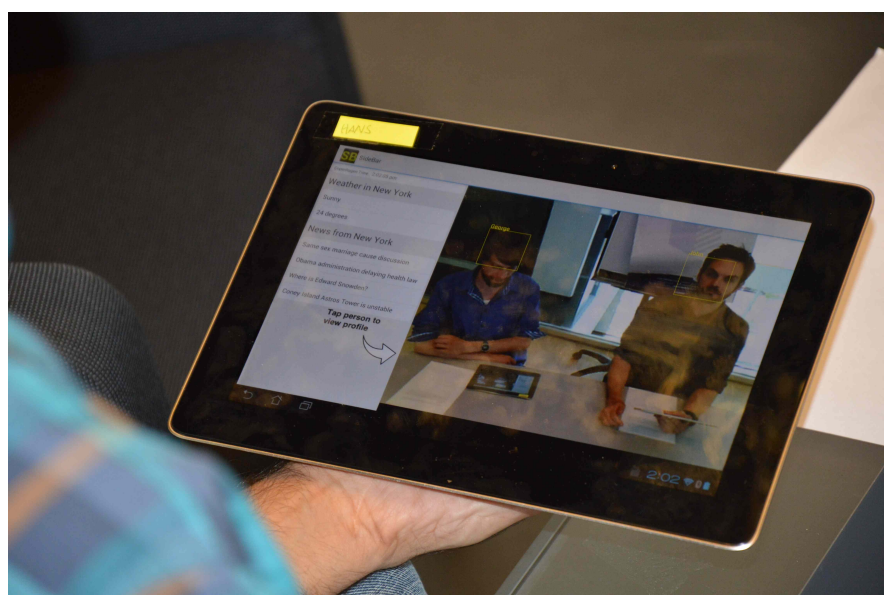

Fig. 2. SIDEBAR extends the video conferencing setup with tablet computers for all meeting participants.

video projection with eye-contact support. TeleHuman [19] uses a cylindrical 3D screen to display life-size video of a person, increasing users sense of social presence and improving the ability to asses gaze and body language cues. LiveMask [28] uses a video setup that presents a movable 3D screen of a persons face upon which live video is projected leading to a more correctly transmitted gaze direction than traditional video setup. More generally, moving and zooming the videoconference camera to suggest movement of participants have been shown to enhance social telepresence of remote actors [29].

In summary, prior research in videoconferencing technologies has focused on enhancing the video meeting by incorporating aspects from collocated meetings, including non-verbal cues and physical presence of remote participants information that can lead to an increase in social awareness [20]. Focus has been on improving the fidelity of the audio-video channel as such, and less on using other channels during videoconferencing to build social relationships and engagement. The latter is the objective of our research and this is approached by integrating face-tracking into the video feed of a regular videoconferencing, and use this to identify and engage with meeting participants, and support ad-hoc backchannel conversations during meetings.

\section{SIDEBAR}

Social aspects among people have a large impact on communication [10] and collaboration [9]. SIDEBAR is a videoconferencing system that focuses on supporting social engagement. As illustrated in Figure 2, the SIDEBAR system extends a traditional videoconferencing system with a tablet computer for each meeting participant.

The purpose of these personal tablets is to provide meeting participants with a tool to recognize remote participants and to engage in backchannel-or sidebar-task-related work, information seeking, and communication. Using face tracking techniques, SIDEBAR provides an interactive mirrored video feed (Figure 3-(1)). Meeting participants can access information about each other via personal profiles (Figure 4-(1)), can learn about the different geographical locations they are located 
in through location profiles (Figure 5), and engage in sidebar conversations using a communication backchannel (Figure 4(2)).

The following scenario describes how SIDEBAR is used in a video meeting.

Each Thursday a video-meeting between a software SME and its distant sister-company is held. The video connection is initiated and everyone logs into SideBar on their tablets. A few participants notice a person at the other site they have not seen before. They use the interactive video feed to navigate to his profile and find out that he has just been assigned to the project. Given recent re-arrangement of teams, he is now the new test manger. The project manager immediately starts the meeting leaving no time for introduction, however, the participants are able to quickly greet the new member using the communication backchannel. As the project manager explains some proposed changes, he has some questions regarding impact and estimates. Using the tablet, he can see which team member has been working on these modules and, since this person is part of the meeting, he can directly address questions to him.

\section{A. System Description}

In the following, the main features of SIDEBAR are described.

Interactive Video Feed. The interactive video feed is the main screen on the SIDEBAR tablet computer (Figure 3-(1)). The user interface augments the video stream from the large conference display with information about each meeting participant. Using the videoconference camera, SIDEBAR tracks people in the meeting room and overlays the video image with a tracking box surrounding each face and adding a name above it. On the tablet, users can tap on the image of a remote participant to visualize his or her personal profile. This helps participants to identify and recognize remote colleagues at a glance, without the need to explicitly look up names in meeting agendas, documents, or by asking local people about who is present.

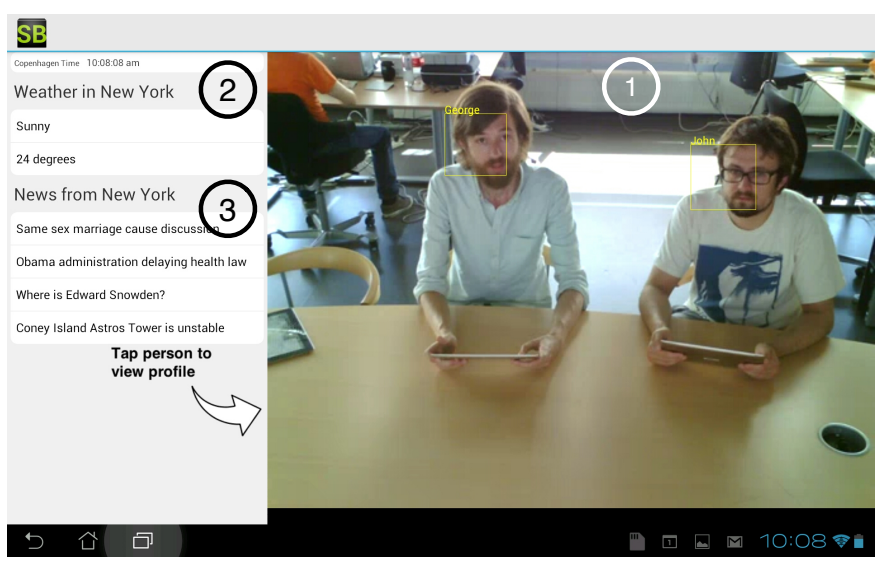

Fig. 3. Interactive video feed on the tablet computer that tracks each participant and display their name (1). Alongside, information about the weather (2) and recent news (3) of the remote site is displayed.
Association Between User and Position. Given the unreliability of face recognition techniques and their need for additional resources (i.e., precise pictures of each person the system should recognize), SIDEBAR only tracks the faces of participants, and the mapping of the person's profile with the correct tracked face is created during the login procedure. When logging in, users are asked to select their name, their geographical location, and the meeting they are participating in. After users have selected these options, a live stream of the local meeting is presented. The user is then asked to select himor her-self in the video stream. Upon selection, the association between the actual user and a tracked face in the stream is created.

Personal Profiles and Team. Each meeting participant has a personal profile in SIDEBAR (Figure 4), which contains both personal and profession information, such as name, age, hometown, profession, education, and interests. This information provides background information and awareness between meeting participants, and can be used to acknowledge similarities between them facilitating the establishment of relationships. Similarly, a team page that provides a side-byside overview of all members of a team is also available.

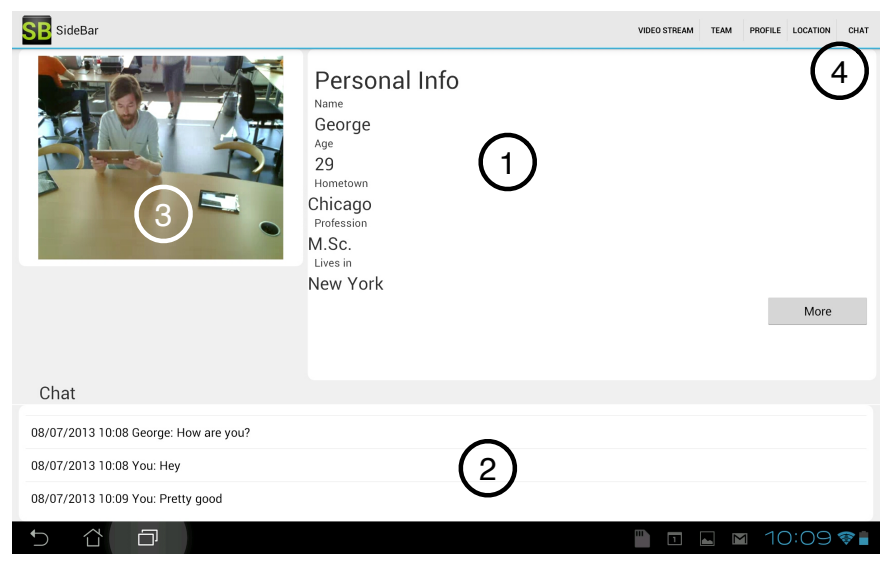

Fig. 4. The personal profile of each meeting participant contains basic personal information (1) and allows to access the communication backchannel ('chat room') with this person (2). Furthermore, the live video feed is displayed (3) and the menu for navigation in the app (4).

Communication Backchannel. One shortcoming of a standard videoconferencing setup is that it provides little opportunities for side conversations among the meeting participants [17]. As discussed above, such side conversations about both professional and more personal matters are important in building and maintaining personal connections in collocated settings. By using the tablet computer, SIDEBAR allows people to engage in backchannel conversations (Figure 4-(2)), hence, providing globally distributed actors with similar opportunities for side conversation.

Local Information. A mundane, yet often observed, obstacle to efficient communication and collaboration across distance is the lack of location information, including weather, time zone, and geography. To help build personal relations, SIDEBAR provides such information in different places. Local information on weather (Figure 3-(2)) and various relevant news feeds (Figure 3-(3)) are visualized in the main screen, and a dedicated display in SIDEBAR providing local information about 
the remote meeting participants and including a description of both the remote office as well as its geographical location is also provided (Figure 5).

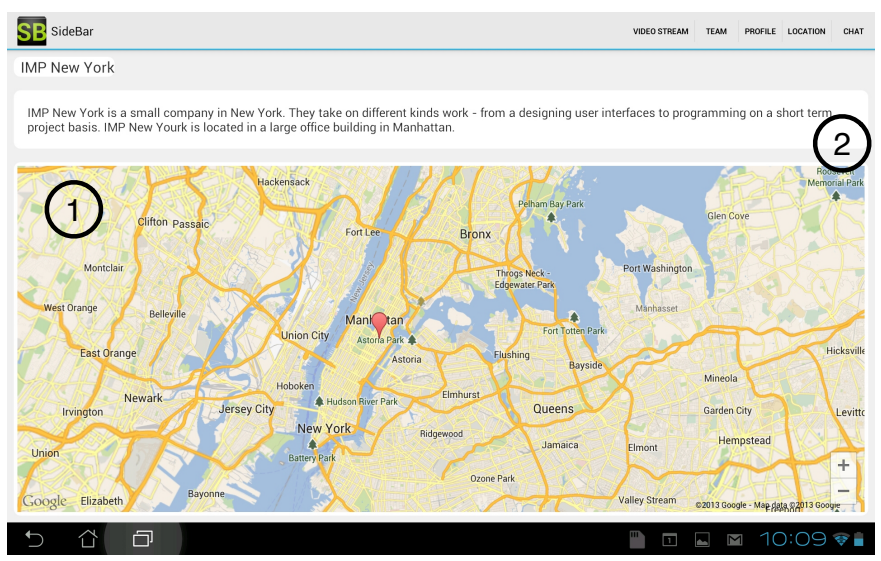

Fig. 5. The location information screen provide simple information about the geographic location of the remote user and the company that he or she is sitting at through a map (1) and a short description (2).

\section{B. Design Methodology}

The SIDEBAR system was designed in a three step process. First, we derived a number of requirements informed by the literature review presented above and field studies reported elsewhere [13], [31]. Next, an initial paper mockup of the SIDEBAR system was designed, which was refined in a usercentered design process with a software SME [32]. Finally, the system was implemented in an initial prototype.

We based our initial design on three overall requirements. First, SIDEBAR should provide users with awareness of each other. Second, SIDEBAR should provide users with opportunities to connect to each other. While technology for communication already exists-email and chat for example are extensively used in collaboration-the opportunity of interaction should exist in conjunction with the awareness of each other. Third, SIDEBAR should integrate with existing technologies and practices. These three overall design requirements formed the basis for adding support for social engagement in a videoconferencing system. This support was added 'on the side' by using tablet computers which allows meeting participants to maintain an awareness of each other, to provide opportunities to connect and communicate, while still being integrated with the video meeting.

We created an initial paper mockup of this design as an input to a design process with a software SME. The company makes extensive use of video meetings with its two offices located in a different parts of the world. The onshore office handles contact with customers while the offshore office handles implementation and test of software. Two workshops focusing on testing the design of the system were conducted, in addition to a series of more informal conversations and discussions of the system design. Each workshop was attended by two researchers and two employees from the company and lasted approximately two hours. The workshops were videotaped for further analysis. Figure 6 shows a snapshot from one of the design workshops.

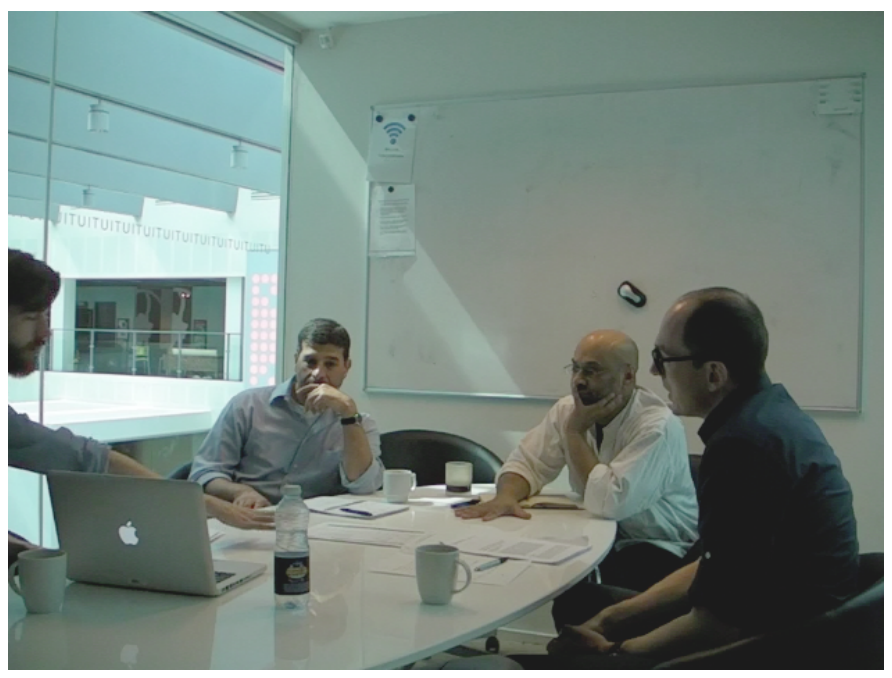

Fig. 6. A snapshot of a design workshop with researchers and industry partners.

These design workshops gave us important insights on the feasibility of the system design and suggestions on how to improve it. First of all, the general idea of providing a SIDEBAR tablet for parallel information seeking, task-based interaction, and communication was well received. The specific UI sketches for looking up information about co-workers, for peer-to-peer communication, and for identifying meeting participants were all considered useful, and the workshop participants provided input for enhancing the UI design of these features. The design study, however, also revealed that some features were missing in the design. Among them, the lack of information-hence, awareness - about the setting and location of remote team members. Information about the local geography, weather, time zone, etc. of the remote place was also considered very useful information for making distributed colleagues more comfortable when contacting each other. Another issue that was raised during the design sessions was that the system should incorporate support for the team aspect of collaboration. The design of the paper prototypes focused on one-to-one connections between people. But considering the role of a person within the team is equally important as a general background information for meeting participants. Finally, we received suggestions for features that were not included in the final design of SIDEBAR. In particular, the participants in the workshop suggested that SIDEBAR should include information about cultural habits associated with the different locations of people. One participant for example, mentioned that small-talk with co-workers about family is common in some parts of the world whereas in other parts, this is seen as inappropriate. While such suggestions indeed are interesting, we decided not to include them in the design of SIDEBAR as such feature should require a thorough investigation of if and how traits associated with cultures can be identified and disseminated.

In summary, on the one hand the user-centered design process confirmed the overall system design of SIDEBAR, while on the other, it provided valuable input for detailed refinements as well as two new important features related to location and team awareness. 


\section{System Architecture \& Implementation}

The SIDEBAR system is composed of several interconnected sub-systems (Figure 7): SideBar App is the tablet application and main user interface to the system; Relation Server is a web server and database system handling data access to all relevant information; the Registration application allows for registration of new users, locations, and meetings; $V L C$ [33] is used to stream video to the tablets; the Tracking Client handles face tracking; finally, Skype [34] is used as the videoconferencing system.

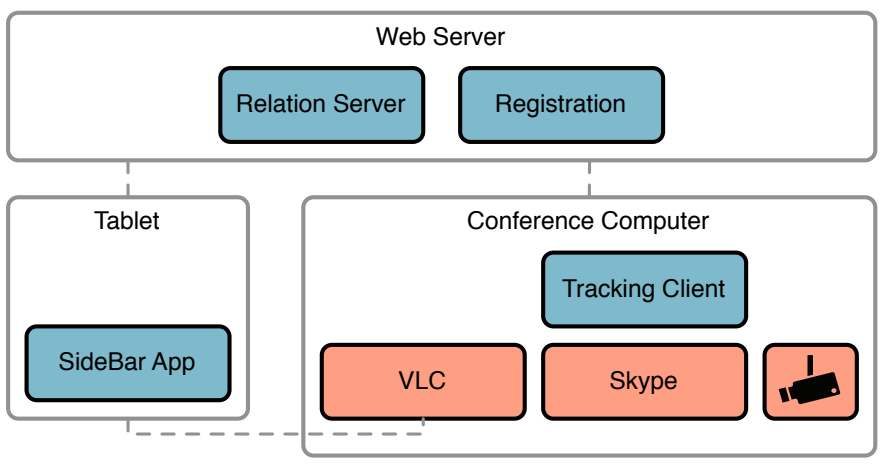

Fig. 7. Overview of the architecture of SIDEBAR. Red boxes represent existing hardware/software, while blue boxes show the novel components of SIDEBAR.

Figure 7 shows an overview of the SIDEBAR system architecture at each meeting location. The conference computer runs the Tracking Client, Skype, and VLC. The Tracking Client performs face tracking, Skype is used for the videoconference, and VLC is used to stream video to the SideBar App. A web server runs the Relation Server and Registration application. The Relation Server is a web application that handles all data access except video. Access to personal profiles, location, meeting, chat, and position information can be achieved using a REST interface of the server. New users, locations or meetings can be added to the system using the Registration application. The SideBar App runs on Android tablets and receives both video streaming over RTSP from the VLC server deployed on the conference computer as well as tracking data, personal and location data, and chat from the Relation Server running on the web server. In the following, we describe the technical details behind the SIDEBAR sub-systems.

Tracking Client. The Tracking Client is a Java application running on the video conference computer implemented via JavaCV [35]. The client determines the position of the head of each meeting participant, and post these to the Relation Server. As previously described, the Tracking Client performs face tracking and not face recognition. Faces are constantly tracked and their positions are regularly sent to the relation server. The association between face positions in the video and actual users is resolved during the login procedure. The Tracking Client constantly updates the position of people, however, it does not allow people to switch seats or leave the meeting and return without logging back in.

Video Streaming. Any videoconferencing setup can be used with SIDEBAR as long as it supports one of the following two requirements; (1) it must expose a camera that VLC and the Tracking Client can access, or (2) it must be possible to place a camera very close to the existing camera to provide a similar video stream to the SIDEBAR system. In our current implementation, Skype is used as the video conferencing technology, and since Skype, VLC, and the Tracking Client are running on the same computer, they all have access to the same camera. Video streaming to the tablets is done using VLC. VLC captures the camera feed and exposes it as an RTP stream playable by the default Android media API. The video is encoded with .h264 and streamed at a resolution of $480 \mathrm{x}$ 360 and a bit rate of $500 \mathrm{Kbps}$.

Relation Server. The Relation server is responsible for data handling of SIDEBAR. The server is implemented in Java using the Java Spring framework and runs in an Apache Tomcat server. The Relation Server stores all information regarding user locations and meetings as well as all position information as tracked by the tracking client and makes this information available through a REST interface to the tablet computers.

SideBar App. SideBAR App is the tablet application and user interface of the SIDEBAR system. SIDEBAR App is implemented in Android 4.0 and is designed to run on a 10.1 inch tablet. SIDEBAR App handles the login procedure, streaming video, backchannel communication, and implements the profiles, location and team profiles.

\section{EVALUATION}

To gather early feedbacks on SIDEBAR, we performed a usability experiment. The key research questions (RQ) addressed by this evaluation were:

RQ1 How useful are the core features of SIDEBAR? Do users perceive SIDEBAR's features useful for improving videoconference meetings, or not?

RQ2 Is SIDEBAR easy to use? Do users perceive SIDEBAR as easy to use, or not?

RQ3 Is the use of SIDEBAR during meetings a distraction? Do users feel distracted when using SIDEBAR during videoconference meetings; does it improve their focus; or do they experience no difference in using it.

These research questions were approached by assessing the so-called 'perceived ease of use' and 'perceived usefulness' of SIDEBAR. Research has shown that there is a strong correlation between user acceptance of a technology and its perceived ease of use and usefulness [36]. In addition to perceived usability and usefulness, we also wanted to explore the notion of 'distraction', since introducing additional devices like the SIDEBAR tablet into a meeting may lead to distraction of the participants.

\section{A. Method}

Given the novelty of the system, we opted to expose study participants to SIDEBAR through a scenario-based approach. This method entails the design of scenarios based on realistic settings exposing participants to complex situations, which would otherwise be hard to observe [37]. Each evaluation session consisted of three phases: a briefing, a scenario, and a debriefing.

In the first phase, participants were welcomed, the SIDEBAR tablet application was demonstrated to them, and they 
were given the opportunity to get accustomed to the application. During this phase the experimenter was present with the participants to answer any questions or concerns regarding both the evaluation procedure as well as the SIDEBAR system.

In the second phase, participants were divided in two groups, were administered a sheet describing the scenario and their role (details below), and were asked to reach their designated position: two rooms were used to simulate two different locations. During this session, they were observed by the experimenter but interactions were kept at a minimum to avoid potential bias.

In the debriefing phase, participants were called back to the initial room and were administered a questionnaire. The questions addressed the three research questions and participants were asked to rate the questions on a 5-point Likert scale $^{1}$ : (RQ1) the usefulness of the main features of SIDEBAR (i.e., interactive video feed, personal profiles, location, communication backchannel, and team information); (RQ2) the extend to which they agreed with the statement "The system is easy to use."; and, (RQ3) the extend to which they agreed with the statement "The use of tablets distracts the video meeting.”. After completing the questionnaire, participants were subject to a semi-structured interview including questions about the general experience with the system. The interview allowed participants to elaborate on their previous answers and to provide detailed comments. Interviews were recorded for further analysis.

Evaluation Scenario. The scenario addressed coordination in distributed software development. A software development scenario was chosen to promote discussion among participants by accommodating the background of the people envisioned as potential candidates for the recruitment, which happened within the SSS department ${ }^{2}$. The scenario focused on a kickoff meeting in which two remote teams of developers had to discuss the design and implementation of a smartphone application. Participants were asked to plan the project using their knowledge about the competences of each other, and divide the work between application development and user interface design. Furthermore, participants were asked to schedule future meetings. Rather then emphasizing the creation of personal connections, the evaluation scenario described a common software scenario, i.e. the kickoff meeting.

The purpose of the study was to investigate whether SIDEBAR was used in connection-making processes without explicitly asking participants to do so. Each participant received a slightly different version of the scenario, which described their specific roles in the scenario. Since participants knew each other, they were all equipped with artificial identities and roles. Participants were asked to enact the character described in the scenario, which required them to 'learn to know each other', which again promoted conversation and the use of the the social features in SIDEBAR.

\section{B. Participants \& Setup}

We recruited a total of seven participants for the evaluation (mean age 32, all male) for two sessions (4 and 3

\footnotetext{
${ }^{1}$ Likert scale parameters: 1 (strongly disagree) to 5 (strongly agree).

${ }^{2}$ SSS department: Software and Systems Section, IT University of Copenhagen, Denmark.
}

\begin{tabular}{lcccccc}
\hline \multicolumn{1}{c}{ Feature } & Min & $Q 1$ & $\tilde{x}$ & Q3 & Max & iqr \\
Interactive video & 4 & 4 & 4 & 5 & 5 & 1 \\
Personal profiles & 3 & 3.25 & 4 & 5 & 5 & 1.75 \\
Location page & 1 & 1.5 & 3 & 3 & 4 & 1.5 \\
Communication backchannel & 4 & 4 & 4 & 5 & 5 & 1 \\
Team Page & 1 & 1.5 & 4 & 4 & 5 & 2.5 \\
$\quad$ Statement & Min & $Q 1$ & $\tilde{x}$ & $Q 3$ & Max & iqr \\
The system is easy to use & 3 & 4 & 4 & 4 & 5 & 0 \\
The use of tablets distracts the & 1 & 1.25 & 4 & 4 & 4 & 2.25 \\
video meeting & & & & & & \\
\hline
\end{tabular}

Fig. 8. Questionnaire result on a 5-point Likert scale. For each feature, the table shows the reported minimum score (Min), the first quartile $(Q 1)$, the median $(\tilde{x})$, the third quartile (Q3), the maximum (Max), and the inter quartile range (iqr).

participants respectively). The participants were a mix of master students, $\mathrm{PhD}$ students, and research assistants. Two compulsory requirements were considered during the selection process related to the experience in software development and the experience in collaborating with distant people using Skype for communication. Besides these requirements, no other inclusion/exclusion criteria were applied. Two meeting rooms were used for the evaluation, each equipped with SIDEBAR. The setup comprised a computer, a large screen, a high resolution webcam with microphone, speakers, and a tablet for each participant. Figure 9 shows a picture from an evaluation session.

\section{Results}

The results of the questionnaire are shown in Table 8. In the following we describe these results in detail.

Interactive Video Feed. The interactive video feed was one of the SIDEBAR features that scored highest $(\tilde{x}=4 ; i q r=1)$. Participants liked the interactive video feed and it was found very useful for navigating. One participant even "felt surprised how useful integrating information and video is" and mentioned that the interactive video feed "added more depth to the video meeting". During the evaluation, we observed that the participants quickly picked up on the names of each other, and used the augmented video feed to reassure themselves about the name of another person before directing a question to this person. Participants were also able to use the interactive video feed to navigate to the personal profiles. Thereby, participants quickly recognized the roles of each other and were able to make the connection between the video image and the person.

Personal Profiles. The personal profile pages were also well received by the participants $(\tilde{x}=4)$, but with some disagreement $(i q r=1.75)$. During the evaluation, the participants actively used the profiles to seek out information about each other. The data they gathered from these profiles were used to direct questions at the right person. The profiles were also used to asses how to divide the work involved in creating the application mentioned in the scenario, thus, aiding them in performing the task described in the scenario. In one session, for example, a participant noted, while referring to the remote site: "you guys are mostly UI designers [...]". This information was then used to argue for a particular division of work.

Location. The location page was the feature that was rated lowest in the evaluation $(\tilde{x}=3 ; i q r=1.5)$. When asked about 


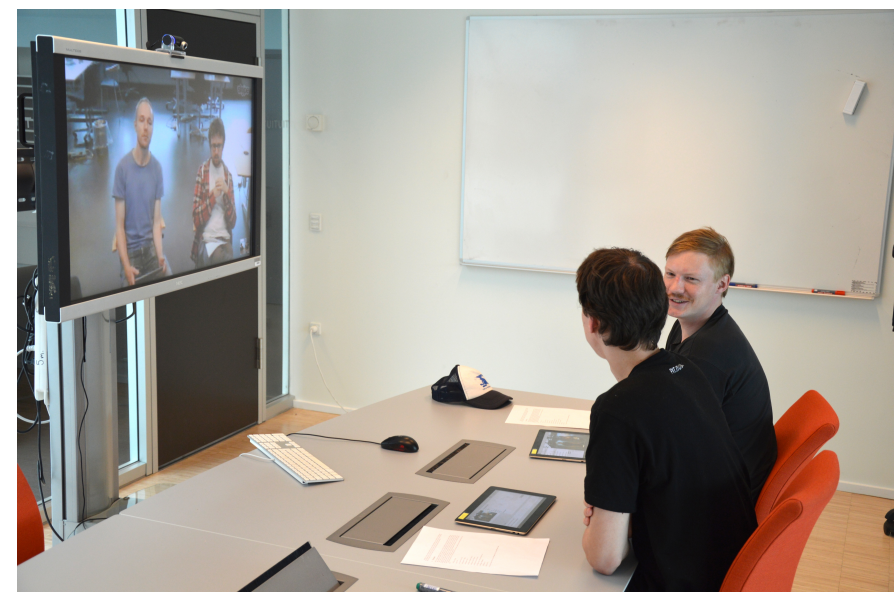

Fig. 9. Evaluation setup. A team of four persons - two at each location-is having a video meeting using SIDEBAR including the larger video display, a camera, and a tablet computer for each meeting participant.

the page after the evaluation, most participants noted that the information was not particularly useful to them. The information provided by the map was, however, used in the evaluation. For example, one participant asked, while referring to the position of the company being located near Central Park in New York: "so your office is near Central Park?". Hence, getting access to local information about the remote site did spark a more informal conversation, thereby building knowledge about the remote participants.

Communication Backchannel. The communication backchannel also scored high in the questionnaire $(\tilde{x}=4$; $i q r=1)$. The participants noted that it provided them with an easy way of sharing textual messages and notes during the meeting. Several participants mentioned that with traditional videoconferencing setups, one-to-one channels for chatting or sharing information are not easily accessible. The participants used the chat actively in the meeting to greet each other and towards the end of the meeting to share relevant information, such as email and web addresses.

Team Profile. There was less agreement on the usefulness of the team information $(\tilde{x}=4 ; i q r=2.5)$. This was also evident from the observations, for some participants used the page actively, while others did not. In the former case, one participant found the team information useful to support the beginning of the meeting in which the teams briefly introduced themselves. In the latter case, another participant found the information in the team page to be redundant, as the same information was available on the profile pages. He noted that it just caused more navigation within the system.

Ease of use. Participant found the system easy to use $(\tilde{x}=4$; $i q r=0)$, and throughout the evaluation, participants were able to navigate within the system without any problems. One remark, however, focused on the fact that the top-menu of the SIDEBAR App is associated with a person, therefore, not accessible on the display showing the video. This caused some confusion as participants were looking for the menu when interacting with the video screen.

Distraction. Participants disagreed on the question of how distracting the tablets were in the video meeting $(\tilde{x}=4 ; i q r=2.25)$.
On the one hand, some participants did not find that the tablets would disturb the meeting and some argued that SIDEBAR would not add more disturbances than the ones already existing. As one participant argued; "it [SIDEBAR] does not disturb more than, for example, printed meeting agendas." Another noted that smart phones and computers are already extensively used during meeting today. On the other hand, two participant noted that eye contact in the video meeting cease when people turn to the tablet. One participant said; "you think you have eye-contact as you see the same video on the tablet-but you don't." Lastly, a participant expressed concern that turning to the tablet felt like turning away from the meeting.

\section{Limitations}

This study is a preliminary evaluation and, as such, presents several limitations posing threats to the validity of its results. The main limitations, which were identified and addressed, are briefly discussed in the following.

First, being a preliminary evaluation, no statistical significance, scalable, or generalizable results were sought, as the key objective was to systematically gather and interpret empirical evidence about the perceived ease of use of the system, the perceived usefulness of its core functionalities, and the user perception of the system as a distraction. With regards to the external validity, even if participants were not practitioners and it could be argued that the sample was not fully representative of the intended population, all participants had software development experience and had experience with remote collaboration. Second, aware of the complexity of assessing collaboration technologies [38], we carefully designed this preliminary evaluation by leveraging established methods (i.e., [37]). Regarding the ecological validity, the scenario was extensively discussed to be as realistic as possible in the simplifications that were applied. This process led us to the decision of simulating a videoconferencing meeting involving three to four people per session separated into two teams physically distributed in different rooms. A hands-on training session was also included before the main scenario to mitigate bias connected to the use of a novel technology. Third, given that our purpose was to gather initial understandings on SIDEBAR, rather than utilizing an established instrument for collecting data, we preferred an ad hoc questionnaire comprising a set of very focused questions. Therefore, the questionnaire used has been designed by the authors, and it was only reviewed and discussed with colleagues knowledgeable and experienced in empirical research. This poses threats to the construct validity; however, the decision allowed us to keep the number of questions to a minimum avoiding lengthly instruments appropriate to more extensive evaluations. Forth, internal validity. Even though all participants satisfied the selection constraints, affiliation with the authors represents a bias. Nonetheless, such recruiting approach is a common practice for preliminary evaluations. Additionally, to avoid experimenter bias, observer/participants interactions were reduced to a minimum by providing participants with a scenario including predesigned roles as described Section IV-A.

Finally, it is worth to notice that no technical validation for the system or its features was conducted. The purpose of the evaluation was purely to get feedback on the design of SIDEBAR, hence, technical aspects were not considered. In a 
future evaluation, the stability and integrity of the system in a realistic setting should be evaluated.

\section{Discussion}

The evaluation of SIDEBAR was designed to provide insights on three research questions. In this section, we discuss these insights.

[RQ1] How useful are the core features of SIDEBAR? The evaluation showed that the participants found SIDEBAR useful and the participants commented on SIDEBAR as being "really helpful" and "the right way to go". In particular, participants liked the linking of video and personal information through the interactive video feed. Furthermore, the communication backchannel was also appreciated. The evaluation, thus, shows promising results for the support of social engagements in video meetings. Nonetheless, some participants found specific features (i.e., the team and location information) less useful compared to the video stream and the communication backchannel. Interestingly, these features were derived from the design workshops we conducted. This points to the possible gap between the design and evaluation; SIDEBAR was designed in collaboration with industry partners, but evaluated with students and researchers. These two aspects of SIDEBAR also contained some redundant information; the team page contained some information that was also available in the profile pages, and the location information was split between the interactive video feed (news and weather) and the location screen (description and map). The information about people and location could be possibly redesigned to be available in one screen to ease navigation.

[RQ2] Is SIDEBAR easy to use? SIDEBAR was found easy to use and participants did not have problems navigating the application, seeking information about each other or using the communication backchannel. A few participants found it confusing that the navigation menu (Figure 4-(4)) was not accessible from the main interactive video screen but only from the profile screens. This suggests that some improvements in terms of navigating in the app should be made.

[RQ3] Is the use of SIDEBAR during meetings a distraction? Participants responded very differently to this question. To really investigate how the introduction of a tablet-based technology like SIDEBAR changes video meetings, a more thorough study should be made, possibly comparing meetings with SIDEBAR to meetings without. For now, it is hard to say whether such a technology would introduce distractions that would disturb the video meetings.

The evaluation of SIDEBAR also pointed out some areas of improvements. Two participants mentioned that the augmented video feed could display even more information than the current implementation offers, including information from the personal profile pages, and highlighting those relevant for the meeting. Also, one participant suggested that the information exposed by SIDEBAR should be available even outside the meeting session. In the current implementation of SIDEBAR, the personal profiles are available only after login-a procedure that requires an ongoing meeting. The suggestion is particularly interesting to consider as in line with our future plans. In fact, on the one hand, social engagements activities are not limited to the meetings per se, and allowing out-ofmeeting usage of SIDEBAR might improve their support. On the other hand, considering that features similar to the ones implemented by SIDEBAR (e.g., personal profiles and communication back channel) are, in some cases, already provided within the ecology of tools used in distributed collaboration projects, integrating existing online social profiles or company chat applications would clearly both facilitate user adoption of the technology as well as increase the chances of finding an industrial partner for performing a field deployment of a company-specific version of SIDEBAR.

\section{DESIGNing For Social Engagement}

Establishing more personal and non-work relations in collaborative settings is an important part of successful collaboration. This is particularly relevant in distributed and global collaboration in which supporting social engagements is more challenging. Therefore — we argue — the design of technologies for distributed collaboration should incorporate support for this social dimension. This section discusses ways of designing for social engagement in distributed collaboration technologies.

We designed and implemented SIDEBAR based on the three design objectives that the system should: support awareness amongst meeting participants, provide them with opportunities to connect to each other, and integrate with existing technologies and practices. These design goals can be generalized to a model of how to design for social engagement in distributed collaboration, as illustrated in Figure 10. Collaborative technologies should provide support for social engagement through: (i) relational context awareness, (ii) relation building and sharing, and (iii) relationship maintenance. In other words, the core design approach is to design for a mutual awareness of relational context, which may trigger relational building and sharing, which again builds and maintains relationships.

\section{A. Relational Context Awareness}

Maintaining an awareness of the nature of relationships between members of an organization is core to social connection making. This happens while overhearing desktop discussions, ad-hoc queries, small exchanges during coffee breaks and in the hallway, and while setting up a meeting. Central to relational context awareness is that people build knowledge about each other and the relationships they are involved in. Design for relational context awareness is evident in architectural design of office space, which is designed with open space where people easily can see and overhear each other, and the office layout is designed so that people easily 'bump into' each other. Similarly, the use of shared artifacts like visible post-it notes, print-outs or drawings on a shared wall allows for the same kind of awareness that collocation brings.

When designing technologies for social engagement, relational context awareness is of great importance. Technologies should seek to provide actors with a sense of awareness about each other. SIDEBAR was designed to support relational context awareness by helping people render relevant relationships visible for others, and for people to be able to monitor the relationships of colleagues. The evaluation of SIDEBAR showed that participants appreciated the awareness 
and the association between video and personal profiles and the information were actively used in the scenarios.

\section{B. Relation Building and Sharing}

A common way to build relationships is the classic team building exercises, company dinners or similar social activities. These activities are all explicitly designed to bring people together within a non-work context. One a more daily basis, informal talks around a shared office space helps establish and maintain connections between people.

Collaborative technologies should seek to provide users with opportunities for building and sharing relations. SIDEBAR provides users with a communication backchannel in video meetings. This channel is accessible from the personal profiles, providing an easy link from the relational context awareness information. As one participant put it, “...this [technology] could replace a kick off meeting" which fits well with the intention of designing for relationship building.

\section{Relationships Maintenance}

Once connections have been established, they need to be maintained and remembered. Often people keep specific artifacts such as pictures, tokens, prizes, diplomas, toys, and award medals from e.g. team building activities as souvenirs and reminders of specific relationships. However, more active involvement is often required to maintain relationships and keep them alive. Social media such as Facebook and Twitter, for example, encourages users to regularly update their online profiles with current information about their doings, interests, and whereabouts etc.

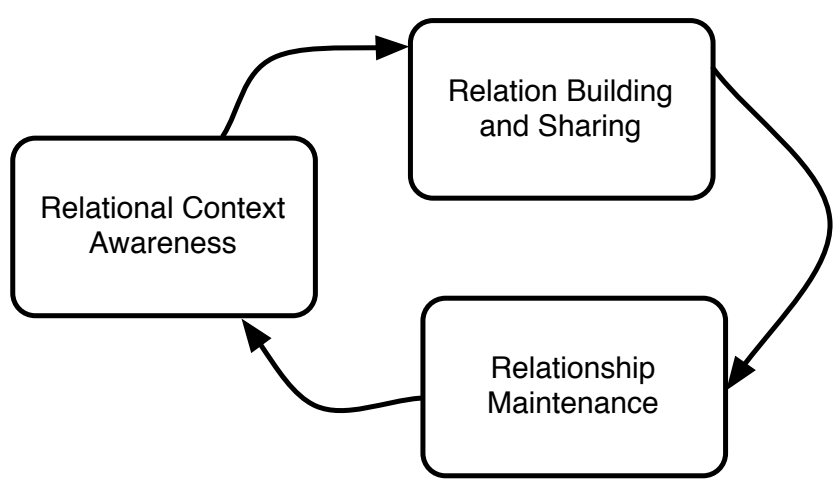

Fig. 10. Design guidelines for social engagement. The core design approach is to design for a mutual awareness of relational context, which may trigger relational building and sharing, which again builds and maintains relationships

In summary, technology should provide relational context awareness, giving access to relationship building and sharing, which in turn promotes relationship maintenance and thereby creating new awareness. If these three processes are supported, a positive spiral of social engagement is achieved in a collaborative setting. By tapping into the design model shown in Figure 10, SIDEBAR is designed to integrate with existing videoconferencing equipment while providing continuous support for social engagement.

\section{CONCLUSION}

In this paper we presented SIDEBAR: a videoconferencing system with a special focus on supporting social engagement. Through the use of face tracking, SIDEBAR offers an interactive mirrored video feed of the ongoing videoconference on tablets, which allows meeting participants to seek information about each other and engage in backchannel conversations. SIDEBAR was designed in a user-centered design process involving a software company implemented in a functional prototype. A preliminary study of SIDEBAR showed that participants found the system easy to use and appreciated both the interactions using an augmented video feed as well as the introduction of a communication backchannel. Based on the design and evaluation of SIDEBAR, we presented three guidelines for the design of technology supporting social engagement revolving around the concepts of relational context awareness, relation building and sharing, and relationship maintenance. In the future, we plan to integrate SIDEBAR into a larger suite of tools for distributed software engineering and evaluate the system in a larger study.

\section{ACKNOWLEDGMENTS}

We would like to thank the participants of the design and evaluation workshops. This research has been funded by the Danish Agency for Science, Technology and Innovation under the project "Next Generation Technology for Global Software Development", \#10-092313.

\section{REFERENCES}

[1] P. J. Hinds and D. E. Bailey, "Out of sight, out of sync: Understanding conflict in distributed teams," Organization Science, vol. 14, no. 6, pp. 615-632, Nov. 2003. [Online]. Available: http://dx.doi.org/10.1287/orsc.14.6.615.24872

[2] J. Herbsleb, "Global software engineering: The future of socio-technical coordination," in Future of Software Engineering, 2007. FOSE '07, May 2007, pp. 188-198.

[3] J. Noll, S. Beecham, and I. Richardson, "Global software development and collaboration: Barriers and solutions," $A C M$ Inroads, vol. 1, no. 3, pp. 66-78, Sep. 2011. [Online]. Available: http://doi.acm.org/10.1145/1835428.1835445

[4] S. Deshpande, I. Richardson, V. Casey, and S. Beecham, "Culture in global software development - a weakness or strength?" in Global Software Engineering (ICGSE), 2010 5th IEEE International Conference on, Aug 2010, pp. 67-76.

[5] G. M. Olson and J. S. Olson, "Distance matters," Hum.-Comput. Interact., vol. 15 , no. 2 , pp. 139-178, sep 2000. [Online]. Available: http://dx.doi.org/10.1207/S15327051HCI1523_4

[6] S. Sarker and S. Sahay, "Implications of Space and Time for Distributed Work: An Interpretive Study of US-Norwegian Systems Development Teams," Eur. J. Inf. Syst., 2004.

[7] J. Herbsleb and A. Mockus, "An empirical study of speed and communication in globally distributed software development," Software Engineering, IEEE Transactions on, 2003.

[8] J. Kotlarsky and I. Oshri, "Social ties, knowledge sharing and successful collaboration in globally distributed system development projects."

[9] S. Kiesler and J. N. Cummings, "What do we know about proximity and distance in work groups? a legacy of research," pp. 57-80, 2002.

[10] B. A. Nardi, "Beyond bandwidth: Dimensions of connection in interpersonal communication," J. Comput.-Supp. Coop. Work, vol. 14, pp. 91-130, 2005.

[11] O. Gotel, V. Kulkarni, M. Say, C. Scharff, and T. Sunetnanta, "Quality indicators on global software development projects: does getting to know you really matter?" Journal of Software: Evolution and Process, vol. 24, no. 2, pp. 169-184, 2012. [Online]. Available: http://dx.doi.org/10.1002/smr.474 
[12] C. A. Fulmer and M. J. Gelfand, "At What Level (and in Whom) We Trust: Trust Across Multiple Organizational Levels," Journal of Management, 2012.

[13] P. Bjørn and L. R. Christensen, "Relation work: Creating socio-technical connections in global engineering," in ECSCW 2011: Proceedings of the 12th European Conference on Computer Supported Cooperative Work, 24-28 September 2011, Aarhus Denmark. Springer London, 2011, pp. 133-152. [Online]. Available: http://dx.doi.org/10.1007/9780-85729-913-0_8

[14] B. Lings, B. Lundell, P. J. Agerfalk, and B. Fitzgerald, "A reference model for successful Distributed Development of Software Systems," in Proceedings of the International Conference on Global Software Engineering, 2007.

[15] N. Bos, N. S. Shami, J. S. Olson, A. Cheshin, and N. Nan, "In-group/out-group effects in distributed teams: An experimental simulation," in Proceedings of the 2004 ACM Conference on Computer Supported Cooperative Work, ser. CSCW '04. New York, NY, USA: ACM, 2004, pp. 429-436. [Online]. Available: http://doi.acm.org/10.1145/1031607.1031679

[16] R. Daft and R. Lengel, "Information Richness: A New Approach to Managerial Behaviour and Organizational Design," Research in Organizational Behaviour, 1984.

[17] E. A. Isaacs and J. C. Tang, "What Video Can and Can'T Do for Collaboration: A Case Study," in Proceedings of the First ACM International Conference on Multimedia, 1993.

[18] R. Vertegaal, I. Weevers, C. Sohn, and C. Cheung, "Gaze-2: conveying eye contact in group video conferencing using eye-controlled camera direction," in Proceedings of the SIGCHI Conference on Human Factors in Computing Systems, ser. CHI '03. New York, NY, USA: ACM, 2003, pp. 521-528. [Online]. Available: http://doi.acm.org/10.1145/642611.642702

[19] K. Kim, J. Bolton, A. Girouard, J. Cooperstock, and R. Vertegaal, "Telehuman: effects of $3 \mathrm{~d}$ perspective on gaze and pose estimation with a life-size cylindrical telepresence pod," in Proceedings of the SIGCHI Conference on Human Factors in Computing Systems, ser. CHI '12. New York, NY, USA: ACM, 2012, pp. 2531-2540. [Online]. Available: http://doi.acm.org/10.1145/2207676.2208640

[20] C. Gutwin, S. Greenberg, and M. Roseman, "Workspace awareness in real-time distributed groupware: Framework, widgets, and evaluation," in Proceedings of HCI on People and Computers XI, ser. HCI '96. London, UK, UK: Springer-Verlag, 1996, pp. 281-298. [Online]. Available: http://dl.acm.org/citation.cfm?id=646683.702625

[21] D. T. Nguyen and J. Canny, "More than face-to-face: empathy effects of video framing," in Proceedings of the SIGCHI Conference on Human Factors in Computing Systems, ser. CHI '09. New York, NY, USA: ACM, 2009, pp. 423-432. [Online]. Available: http://doi.acm.org/10.1145/1518701.1518770

[22] P. Slovák, P. Novák, P. Troubil, P. Holub, and E. C. Hofer, "Exploring trust in group-to-group video-conferencing," in CHI '11 Extended Abstracts on Human Factors in Computing Systems, ser. CHI EA '11. New York, NY, USA: ACM, 2011, pp. 1459-1464. [Online]. Available: http://doi.acm.org/10.1145/1979742.1979791

[23] D. Grayson and A. Anderson, "Perceptions of proximity in video conferencing," in CHI '02 Extended Abstracts on Human Factors in Computing Systems, ser. CHI EA '02. New York, NY, USA: ACM, 2002, pp. 596-597. [Online]. Available: http://doi.acm.org/10.1145/506443.506501

[24] T. Jenkin, J. McGeachie, D. Fono, and R. Vertegaal, "eyeview: focus+context views for large group video conferences," in CHI '05 Extended Abstracts on Human Factors in Computing Systems, ser. CHI EA '05. New York, NY, USA: ACM, 2005, pp. 1497-1500. [Online]. Available: http://doi.acm.org/10.1145/1056808.1056950

[25] C. Kuster, T. Popa, J.-C. Bazin, C. Gotsman, and M. Gross, "Gaze correction for home video conferencing," ACM Trans. Graph., vol. 31, no. 6, pp. 174:1-174:6, nov 2012. [Online]. Available: http://doi.acm.org/10.1145/2366145.2366193

[26] C. Harrison and S. Hudson, "Pseudo-3d video conferencing with a generic webcam," in Multimedia, 2008. ISM 2008. Tenth IEEE International Symposium on, 2008, pp. 236-241.

[27] K.-I. Okada, F. Maeda, Y. Ichikawaa, and Y. Matsushita, "Multiparty videoconferencing at virtual social distance: Majic design," in Proceedings of the 1994 ACM conference on Computer supported cooperative work, ser. CSCW '94. New York, NY, USA: ACM, 1994, pp. 385-393. [Online]. Available: http://doi.acm.org/10.1145/192844.193054

[28] K. Misawa, Y. Ishiguro, and J. Rekimoto, "Livemask: a telepresence surrogate system with a face-shaped screen for supporting nonverbal communication," in Proceedings of the International Working Conference on Advanced Visual Interfaces, ser. AVI '12. New York, NY, USA: ACM, 2012, pp. 394-397. [Online]. Available: http://doi.acm.org/10.1145/2254556.2254632

[29] H. Nakanishi, K. Kato, and H. Ishiguro, "Zoom cameras and movable displays enhance social telepresence," in Proceedings of the SIGCHI Conference on Human Factors in Computing Systems, ser. CHI ' 11. New York, NY, USA: ACM, 2011, pp. 63-72. [Online]. Available: http://doi.acm.org/10.1145/1978942.1978953

[30] G. Aranda, A. Vizcaí andno, R. Palacio, and A. Morá andn, "What Information Would You Like to Know about Your Co-worker? A Case Study," in Global Software Engineering (ICGSE), 2010 5th IEEE International Conference on, 2010.

[31] L. R. Christensen, R. E. Jensen, and P. Bjørn, "Relation work in collocated and distributed collaboration," in Proc. COOP 2014. Springer, 2014.

[32] D. A. Norman and S. W. Draper, "User centered system design," New Perspectives on Human-Computer Interaction, L. Erlbaum Associates Inc., Hillsdale, NJ, 1986.

[33] VLC, "http://www.videolan.org/." [Online]. Available: http://www.videolan.org/

[34] Skype, "http://www.videolan.org/." [Online]. Available: http://www.videolan.org/

[35] JavaCV, "https://code.google.com/p/javacv/." [Online]. Available: https://code.google.com/p/javacv/

[36] F. D. Davis, "Perceived usefulness, perceived ease of use, and user acceptance of information technology," MIS Quarterly, vol. 13, no. 3, pp. 319-339, September 1989.

[37] G. Convertino, D. C. Neale, L. Hobby, J. M. Carroll, and M. B. Rosson, "A laboratory method for studying activity awareness," in Proceedings of the third Nordic conference on Human-computer interaction, 2004.

[38] J. Whitehead, "Collaboration in software engineering: A roadmap," Future of Software Engineering (FOSE '07), 2007. 